\title{
The use of a combined process of surfactant-aided soil washing and coagulation for
} PAH-contaminated soils treatment

\author{
R. López-Vizcaíno, C. Sáez, P. Cañizares, M.A. Rodrigo* \\ Department of Chemical Engineering, University of Castilla-La Mancha, Enrique Costa \\ Building, Av. Camilo José Cela, nº 12, 13071 Ciudad Real, Spain.
}

\begin{abstract}
Polycyclic aromatic hydrocarbons (PAHs) persist in soils due to their low volatility, low water solubility and low biodegradability, all of which make it difficult to remove this type of compound from soils. The work described here involved the study of a combined surfactant-aided soil washing (SASW) process and coagulation treatment, using iron and aluminium salts, to remediate a low-permeability PAH-polluted soil. Phenanthrene was selected as the model PAH and three different types of surfactants (anionic, cationic and non-ionic) were used as washing agents. The results show that the anionic surfactant is the most effective washing fluid because efficiencies higher than $90 \%$ can be achieved. Non-ionic and cationic surfactant efficiencies were $70 \%$ and $30 \%$, respectively. In addition, only the anionic wastewater can be satisfactorily treated with this technology, with COD removals greater than $90 \%$ achieved. Variation of $\mathrm{pH}$, zeta-potential and the dose of aluminium required seem to indicate that a chargeneutralization mechanism is the main process involved in the emulsion break-up obtained in the treatment of aqueous surfactant wastes. In addition, the effects of surfactant concentration and that of the $\mathrm{pH}$ of the soil-washing wastewater seem to have a greater influence on the performance of the coagulation process.
\end{abstract}

Keywords: clayed soil, surfactant, soil washing, phenanthrene, coagulation. 
* Corresponding author. Tel.: +34 902204100ext3411; fax: +34 926295256.

E-mail address: Manuel.Rodrigo@uclm.es. 


\section{Introduction}

Polycyclic aromatic hydrocarbons (PAHs) are a group of more than 100 chemically different substances that are generally composed of non-polar molecules formed by two or more benzene rings [1]. For this reason, these compounds are classified as hydrophobic organic compounds. These substances are formed during the incomplete combustion of coal, oil, diesel or gasoline, and other organic substances. Normally, these materials enter the environment from several sources: the gases emitted by engines during combustion of gasoline and especially of diesel, the combustion of wood or coal, and from sites associated with industries such as gas production, the refinement of oil and the manufacture of wood. PAHs are an important class of pollutants since some compounds have been classed as carcinogenic, mutagenic and teratogenic [2-5]. Moreover, they are very persistent in soils and sediments due to properties such as low volatility, low water solubility and low biodegradability. These characteristics make it very difficult to remove this type of compounds from soils [6, 7].

Surfactant-aided soil washing (SASW) is one of the standard technologies in the remediation of soils polluted with PAHs [8-11]. This approach involves washing the soils with an aqueous surfactant solution with the aim of enhancing the water solubility of the PAHs and forming $\mathrm{O} / \mathrm{W}$ emulsions with micro-drops of $\mathrm{PAH}$. The resulting emulsions can subsequently be treated by a wastewater treatment technology such as: electrochemical oxidation, ozonation, biological treatment, etc. [12,13]. In this way PAHs are removed from the soils and the pollution is transferred to the washing fluid. 
At present, one of the most relevant technologies for the treatment of $\mathrm{O} / \mathrm{W}$ emulsions is coagulation. This method involves destabilization of the micro-drops of organics by the action of a chemical reagent (usually an iron or aluminium species), which leads to the break-up of the emulsion and the formation of two phases that can be easily separated.

The objective of the work described here was to study the combination of a SASW process with a coagulation process to remediate a low-permeability phenanthrene-polluted soil (kaolinite). In this study two partial objectives were set: firstly, to compare the effectiveness of the SASW process with low-permeability soils polluted with phenanthrene using three different types of surfactants as washing reagents (anionic, cationic and non-ionic) and, secondly, to treat the effluents produced in the SASW process by coagulation using iron and aluminium salts.

\section{Materials and methods}

Kaolinite was selected as the model for a clay soil in this work. This material is not reactive and it has low hydraulic conductivity, low cation exchange capacity and no organic content. The physico-chemical properties of this soil are shown in Table 1. Phenanthrene (97\%) was selected as a model PAH and this compound was obtained from Merck. 


\subsection{Preparation of simulated soil}

Samples of polluted soil were prepared by dissolving phenanthrene in acetone and then mixing this phenanthrene/acetone solution with the kaolinite. The spiked clay was aerated during one day to favour evaporation of the acetone and in this way the phenanthrene was homogeneously distributed on the clay surface. This method has been described in the literature by different researchers [14-17].

\subsection{Surfactant-aided soil washing}

Surfactant-aided soil washing tests were carried out in a stirred bench-scale tank operated in discontinuous mode. The tank volume was $2000 \mathrm{~cm}^{3}$. Low-permeability soil (135 g) polluted with $500 \mathrm{mg}$ phenanthrene $\mathrm{kg}^{-1}$ of soil and $1800 \mathrm{~cm}^{3}$ of washing solution (containing deionised water and $10 \mathrm{~g} \mathrm{dm}^{-3}$ of surfactant) were mixed in the reactor for 6 hours at a stirring speed of $120 \mathrm{rpm}$ [14]. The same tank then acts as a settler (during 24 hours) to separate the soil from the effluent generated during the soilwashing process. These effluents consisted of aqueous mixtures of phenanthrene and surfactants with a very high COD. The phenanthrene removal percentage (PRP) was calculated using Eq. (1), where $C_{f}$ is the outstanding phenanthrene concentration and $C_{o}$ is the initial concentration in soil samples.

$$
\operatorname{PRP}(\%)=\left(1-\frac{C_{f}}{C_{o}}\right) \cdot 100
$$


Three types of surfactants were used to formulate the soil-washing solutions: Sodium dodecyl sulphate (SDS) as a model anionic surfactant, alkylbenzyldimethylammonium chloride (ABDMA) as a model cationic surfactant and polyoxyethylene sorbitan monooleate (Tween 80 ) as a model non-ionic surfactant. The properties of these compounds are given in Table 2 .

\subsection{Coagulation of soil-washing wastewater}

Discontinuous chemical coagulation experiments were carried out in a standard jar test experimental set-up coaple with a thermostatic bath (J.P. SELECTA, Spain). In Each experiment, a fixed amount of coagulant solutions $\left(134 \mathrm{~g} / \mathrm{l} \mathrm{AlCl} 3 \cdot 6 \mathrm{H}_{2} \mathrm{O}\right.$ or $240 \mathrm{~g} / \mathrm{l}$ $\mathrm{FeCl}_{3}$ ) was added to $150 \mathrm{ml}$ of soil-washing wastewater. This solution was vigorously and slowly stirred during 3 and 15 minutes respectively, and after that it was left during 60 minutes to allow the sedimentation of the solid particles.

\subsection{Analyses}

To quantify the amount of phenanthrene present in the soil, it is used an L-S extraction process. It is carried out in Eppendorf tubes $(15 \mathrm{ml})$ and using hexane as solvent (ratio polluted soil/solvent $=0.2 \mathrm{w} / \mathrm{w})$. Both phases was vigorously stirred in a VV3 VWR multi-tube and subsequent phase separation was accelerated by the use of a centrifuge rotor angular CENCOM II P-elite. To determine the phenanthrene concentration in liquid phase an L-L extraction process was used also. It is carried out in bottles (100 ml) and using hexane as solvent also (ratio PHE solution/solvent $=0.22 \mathrm{v} / \mathrm{v}$ ). Both phases 
were slowly stirred in an orbital shaker $(100 \mathrm{rpm})$ during $5 \mathrm{~h}$ (operation time required to attain the steady state).

In both cases, the concentration of phenanthrene in the resulting liquid extract phase was determined by UV-visible spectrometry (Shimadzu UV-1603). The characteristic absorbance peaks of phenanthrene in the UV-visible spectrum were at 346,338 and 330 nm. The absorbance at these three wavelengths was used to quantify the phenanthrene concentration. The standard deviation of this determination is lower than $5 \%$.

To check the mass balance of the system and to verify the quality of the analytical metahology used, it was used the following methodoly. Firstly, the total initial mass of the soil was divided in 4 portions and each portion was subdivided in other 3 portions. Secondly, it was measured the initial PHE concentration of each portion of soil. To do this, PHE was extracted with hexane (as it was explained previously). Thirdly, it was carried out the washing process of each portion with anionic surfactant in order to remove the PHE from the soil. After that, the concentration of PHE of both phases (liquid and solid) was measured. To do this, the PHE was extracted with hexane (as it was explained previously). Finally, the error of the global process can be calculated using the equation 2. Table 3 summarizes the results obtained in the study. As it can the average error was lower than $10 \%$. This value is comparable with data reported in literature $[9,10,13]$.

$$
\text { Error }=100 \cdot\left(\frac{P H E_{\text {Balance }}-P H E_{\text {initialinøil }}}{P H E i_{\text {nitialinsoll }}}\right)
$$


The removal of washing effluents was evaluated by monitoring the chemical oxygen demand using a HACH DR2000 analyzer [18]. Zeta potential was also measured for the clarified liquid using a Zetasizer Nano ZS (Malvern, UK). The turbidity measurement was carried out in a 115 VELP SCIENTIFIC turbidimeter, using the Nephelometric Method [18]). This instrument measures the intensity of light scattered 90 degrees from the path of incident light.

\section{Results and discussions}

\subsection{Surfactant-aided Soil-Washing (SASW) Process}

The dynamics of the surfactant-aided soil-washing processes are represented in Fig. 1. In this graph, an increase in the phenanthrene removal percentage (PRP) values with the washing time indicates that the surfactant solutions could extract more phenanthrene from spiked soil. It can be observed that in all three cases the PRP increases sharply during the first hour and then a constant value is reached. At this point, the system has reached stationary conditions and further washing times are not able to increase the PRP. Taking into account these results, a washing time of 6.0 hours was selected to study the steady-state influence of parameters in the SASW process. Another important observation that can be made from Fig. 1 is the higher efficiency of the anionic surfactant in the removal of phenanthrene from soils. Thus, for the same surfactant dose, the soil-washing with the anionic surfactant (around $90 \%$ of PRP) shows markedly better results than the non-ionic surfactant (around $70 \%$ of PRP) and, furthermore, it shows twice the efficiency obtained by the cationic surfactant (around 35 $\%$ of PRP). These results are in agreement with those reported in literature [13,19-23]. 
However, it is difficult to make a direct comparison as the extraction percentages removal and thus the efficiency of the process depend, among other factors, on the ratio surfactant/pollutant and fluid-dynamic conditions.

The effect of the surfactant dose in the steady-state PRP obtained during the SASW process is shown in Fig. 2. For the ionic surfactants, an increase in the dose leads to an increase in the efficiency of the SASW process. The changes are more significant in the case of the cationic surfactant, although the steady-state PRPs obtained are smaller than those observed with the anionic surfactant. This is in agreement with data reported in literature [19-22]. According to these works, it can be assumed that it is related to the higher solubility of PHE in high concentrated surfactant solutions. On the other hand, the results obtained with the non-ionic surfactant are opposite to those described above: an increase in the surfactant dose (until $2 \% \mathrm{wt}$ ) seems to have a detrimental effect on the results obtained in the SASW process. At the light of the actual knowledge, this behaviour is difficult to explain although it may be related to the absorption of surfactant-contaminant group at the soil surface [23].

The effect of the pollutant concentration on the washing capacity of the SASW process is represented in Fig. 3. Within the range studied (400-2000 $\mathrm{mg} \mathrm{kg}^{-1}$ ) it can be observed that the higher the level of pollution in the soil, the higher the level of phenanthrene removed by soil washing. In all cases the anionic surfactant was the most efficient, with removal of pollutants over $90 \%$. 


\subsection{Treatment of soil-washing wastewaters with coagulation technologies}

The wastewaters produced in an SASW process consist of an emulsion of phenanthrene in water stabilized by the surfactants [24]. In addition, wastewater can also contain particles of soil dragged in during the washing process. Consequently, the effluents consist simultaneously of a colloidal suspension and an $\mathrm{O} / \mathrm{W}$ emulsion. Aggregation of the micro-drops of phenanthrene and the micro-particles is not favoured due to the existence of superficial electrical charge in these particles. Therefore, in order to obtain an effective process for the treatment of this wastewater a stage is required in which the destabilization of the emulsion and colloidal suspension is promoted by the addition of coagulants [25].

The influence of the dose of aluminium on the removal of COD during the coagulation of the three types of wastewaters is represented in Fig. 4. It can be observed that the coagulation process is only able to destabilize the wastewater polluted with anionic surfactant and is completely inefficient for the other two wastes. The dose of aluminium required is not very high for the concentration of pollutant, and this suggests a charge-neutralization mechanism to explain the emulsion break-up. This coagulation mechanism was confirmed by the $\mathrm{pH}$ and z-potential measurements shown in Fig. 5. The $\mathrm{pH}$ decreases to 3.2 due to the acidic properties of the aluminium chloride added and the z-potential increases to 0 for the same dose for which the emulsion break-up is observed (10 mmol Al $\left.\mathrm{ml}^{3+} \mathrm{dm}^{-3}\right)$.

The effect that the surfactant concentration has on the washing-water and the initial phenanthrene concentration in the soil has on the efficiency of the coagulation of 
the wastewaters produced in the SASW process are shown in Fig. 6. In all cases coagulation enables COD removal greater than 90\%, although the doses required depend on the concentration of phenanthrene and on the concentration of surfactant. The effect of these two parameters is different in terms of importance, with the surfactant concentration being more significant. Thus, the dose of aluminium depends strongly on the concentration of surfactant because an increase in the concentration of surfactant from 1 to $2 \%$ almost doubles the required break-up dose. In contrast, an increase in the concentration of phenanthrene only leads to a slight increase in the dose of aluminium required. This behaviour is expected because the coagulant reagent only interacts with the sulphate groups of the surfactants and the phenanthrene concentration only influences the size of the micro-drops of the emulsion, which can also be influenced by many other parameters in the SASW process such as stirring rate or surfactant/phenanthrene ratio. Conversely, as can be observed in Fig. 7, the concentration of coagulant reagent required to attain turbidity values below $10 \mathrm{NTU}$ does not seem to depend strongly on the presence of phenanthrene.

The effect of a modification in the initial $\mathrm{pH}$ of the SASW wastewater on the results of the coagulation process is represented in Fig. 8. It can be observed that this change does not influence the final results of the emulsion break-up, but it does have a marked influence on the required dose of reagents. The acidic properties of aluminium chloride always lead to a low $\mathrm{pH}$ of the coagulation system and this change helps to neutralize negative charges on the micro-drops, as evidenced by changes in the $\mathrm{z}$ potential. In the case of the wastewater modified to acidic $\mathrm{pH}$, the $\mathrm{pH}$ does not change appreciably during the treatment and the z-potential is around the isoelectric point. The $\mathrm{pH}$ could be the most important parameter in a coagulation process as both coagulant 
species and coagulation mechanisms are dependent on this factor. The results obtained indicate that monomeric cationic species (those that prevail at this $\mathrm{pH}$ range) are the only effective species in the coagulation of this type of wastewater.

The effects of the type of reagent used on the results of the coagulation process are shown in Fig. 9. Numerous studies have been published in which the influence of the type of coagulant used on coagulation processes have been evaluated [26-29] and this is the result of their significant influence on the coagulation mechanisms. The most widely used coagulants in these processes are salts of aluminium or iron. In this study tests using aluminium, iron, and mixtures of the two metals as coagulants were carried out. It was observed that the performance obtained in the process (expressed in terms of both COD and turbidity removal) does not depend on the type of coagulant used. This finding suggests that both metals act with the same coagulation mechanism.

\section{Conclusions}

Surfactant-aided soil washing (SASW) gives good results for the remediation of low-permeability soils polluted with phenanthrene. Of the three different types of surfactants used as washing reagents, the anionic surfactant was the most efficient and gave pollutant removal percentages over $90 \%$ (double the efficiency obtained with the cationic surfactant). The coagulation process appears to be a good alternative for the treatment of SASW-wastewater, which consists of an emulsion of phenanthrene in water stabilized by the surfactants. The dose of aluminium required is not very high and a charge-neutralization mechanism can explain the emulsion break-up obtained in the treatment of surfactant aqueous wastes. On the other hand, the effect of surfactant 
concentration on the soil-washing wastewater seems to be more significant in the performance of the coagulation process than phenanthrene concentration. The coagulant reagent interacts mainly with the sulphate groups of the surfactant while phenanthrene only influences the size of the microdrops. The results obtained do not depend on the type of coagulant used (iron or aluminium salts).

\section{Acknowledgements}

Financial support from the Spanish government through project CTM2007-60472 is gratefully acknowledged. 


\section{References}

1. D.W. Connell, Basic Concepts of Environmental Chemistry, Lewis Publishers, New York, 1997.

2. K.R. Reddy, P.R. Ala, S. Sharma, S.N. Kumar, Enhanced electrokinetic remediation of contaminated manufactured gas plant soil, Eng. Geol. 85 (2006) 132-146.

3. P. Gerde, B.A. Muggenburg, M. Lundborg, A.R. Dahl, The rapid alveolar absorption of diesel soot-adsorbed benzo[a]pyrene: bioavailability, metabolism and dosimetry of an inhaled particle-borne carcinogen, Carcinogenesis 22 (2001) 741749.

4. P-J. Tsai, H-Y. Shieh, W-J. Lee, S-O. Lai, Health-risk assessment for workers exposed to polycyclic aromatic hydrocarbons (PAHs) in a carbon black manufacturing industry, Sci. Total Environ. 278 (2001) 137-150.

5. A.B. Ribeiro, J.M. Rodríguez-Maroto, E.P. Mateus, H. Gomes, Removal of organic contaminants from soils by an electrokinetic process: the case of atrazine.: Experimental and modelling, Chemosphere, 59 (2005) 1229-1239.

6. D.G. Williamson, C.L. Raymond, Y.C. Kimura, Release of chemicals from contaminated soils, J. Soil Contam. 7 (1998) 543-558.

7. A.W. Hatheway, Geoenvironmental protocol for site and waste characterization of former manufactured gas plants; worldwide remediation challenges in semi volatile organic wastes, Eng.Geol. 64 (2002) 317-338.

8. K. Elgh-Dalgren, Z. Arwidsson, A. Camdzija, R. Sjöberg, V. Ribé, S. Waara, B. Allard, T. von Kronhelm, P. A.W. van Hees, Laboratory and pilot scale soil washing of PAH and arsenic from a wood preservation site: Changes in concentration and toxicity, J. Hazard. Mater. 172 (2009) 1033-1040. 
9. R.D. Villa, A. G. Trovó, R. F. Pupo Nogueira, Soil remediation using a coupled process: soil washing with surfactant followed by photo-Fenton oxidation, J. Hazard. Mater. 174 (2010) 770-775.

10. L. A. Bernardez, Dissolution of polycyclic aromatic hydrocarbons from a nonaqueous phase liquid into a surfactant solution using a rotating disk apparatus, Colloids Surf., A. 320 (2008) 175-182.

11. W. Chu, K. H. Chan, The mechanism of the surfactant-aided soil washing system for hydrophobic and partial hydrophobic organics, Sci. Total Environ. 307 (2003) 83-92.

12. P. Haapea, T. Tuhkanen, Integrated treatment of PAH contaminated soil by soil washing, ozonation and biological treatment, J. Hazard. Mater. 136 (2006) 244-250.

13. J. Gómez, M.T. Alcántara, M. Pazos, M.A. Sanromán, Remediation of polluted soil by a two-stage treatment system: Desorption of phenanthrene in soil and electrochemical treatment to recover the extraction agent , J. Hazard. Mater. 173 (2010) 794-798

14. M-C. Chang, C-R. Huang, H-Y. Shu, Effects of surfactants on extraction of phenanthrene in spiked sand, Chemosphere, 41 (2000) 1295-1300.

15. J-Y. Park, H-H. Lee, S-J. Kim, Y-J. Lee, J-W. Yang, Surfactant-enhanced electrokinetic removal of phenanthrene from kaolinite, J. Hazard. Mater. 140 (2007) 230-236.

16. M. T. Alcántara, J. Gómez, M. Pazos, M.A. Sanromán, PAHs soil decontamination in two steps: Desorption and electrochemical treatment, J. Hazard. Mater, 166 (2009) 462-468. 
17. M.T. Alcántara, J. Gómez, M. Pazos, M.A. Sanromán, Combined treatment of PAHs contaminated soils using the sequence extraction with surfactantelectrochemical degradation, Chemosphere, 70 (2008) 1438-1444.

18. APHA, AWWA, WPCF. Standard Methods for the Examination of Water and Wastewater, 20th ed.; Clesceri, L. S., Greenberg, A. E., Eaton, A. D., Franson, M. A. H., Eds.; American Public Health Association: Washington, DC, 1989.

19. J. Gómez, M. T. Alcántara, M. Pazos, M. A. Sanromán, Soil washing using cyclodextrins and their recovery by application of electrochemical technology, Chem. Eng. J. 159 (2010) 53-57.

20. C.K. Ahn, Y.M. Kim, S.H. Woo, J.M. Park, Soil washing using various nonionic surfactants and their recovery by selective adsorption with activated carbon, J. Hazard. Mater., 154 (2008) 153-160.

21. A. Petitgirard, M. Djehiche, J. Persello, P. Fievet, N. Fatin-Rouge, PAH contaminated soil remediation by reusing an aqueous solution of cyclodextrins, Chemosphere, 75 (2009) 714-718.

22. W. Zhou, L. Zhu, Enhanced soil flushing of phenanthrene by anionic-nonionic mixed surfactant, Water Res. 42 (2008) 101-108.

23. S. Deshpande, B. J. Shiau, D. Wade, D. A. Sabatini, J. H. Harwell, Surfactant selection for enhancing ex situ soil washing, Water Res. 33 (1999) 351-360.

24. USEPA. A resource for MGP site characterization and remediation. EPA/542-R-00005, Washington, DC, 2000.

25. T. Chatterjee, S. Chatterjee, D.S. Lee, M.W. Lee, S.H. Woo, Coagulation of soil suspensions containing nonionic or anionic surfactants using chitosan, polyacrylamide, and polyaluminium chloride, Chemosphere, 75 (2009) 1307-1314. 
26. A. Jain, V.K. Sharma, O.S. Mbuya, Removal of arsenite by $\mathrm{Fe}(\mathrm{VI}), \mathrm{Fe}(\mathrm{VI}) / \mathrm{Fe}(\mathrm{III})$, and $\mathrm{Fe}(\mathrm{VI}) / \mathrm{Al}(\mathrm{III})$ salts: Effect of pH and anions, J. Hazard. Mater. 169 (2009) 339344.

27. I. Zongo, A.H. Maiga, J. Wéthé, G. Valentin, J-P. Leclerc, G. Paternotte, F. Lapicque, Electrocoagulation for the treatment of textile wastewaters with $\mathrm{Al}$ or $\mathrm{Fe}$ electrodes: Compared variations of COD levels, turbidity and absorbance, J. Hazard. Mater. 169 (2009) 70-76.

28. E. Barbot, P. Dussouillez, J.Y. Bottero, P. Moulin, Coagulation of bentonite suspension by polyelectrolytes or ferric chloride: Floc breakage and reformation, Chem. Eng. J. 156 (2010) 83-91.

29. P. Cañizares, C. Jiménez, F. Martínez, C. Sáez, M.A. Rodrigo, Study of the Electrocoagulation Process Using Aluminum and Iron Electrodes, Ind. Eng. Chem. Res. 46 (2007) 6189-6195. 


\section{Table 1}

Physico-chemical properties of kaolinite.

\begin{tabular}{lr|lr}
\hline \multicolumn{2}{c|}{ Mineralogy } & \multicolumn{2}{c}{ Particle size distribution (\%) } \\
\hline Kaolinite & $100.00 \%$ & Gravel & 0 \\
$\mathrm{Fe}_{2} \mathrm{O}_{3}$ & $0.58 \%$ & Sand & 4 \\
$\mathrm{TiO}_{2}$ & $0.27 \%$ & Silt & 18 \\
$\mathrm{CaO}$ & $0.10 \%$ & Clay & 78 \\
\cline { 2 - 2 } & $0.75 \%$ & Specific gravity & 2.6 \\
$\mathrm{~K}_{2} \mathrm{O}$ & $52.35 \%$ & Hydraulic conductivity (cm/s) & $1 \times 10^{-8}$ \\
$\mathrm{Al}_{2} \mathrm{O}_{3}$ & $34.50 \%$ & Organic content (\%) & 0 \\
Others & $11.42 \%$ & pH & 4.9 \\
\hline
\end{tabular}

Table 2

Properties of Surfactants.

\begin{tabular}{|c|c|c|c|c|c|c|}
\hline Surfactant & Type & Formula & MW & $\mathrm{CMC} / \mathrm{gl}^{-1}$ & Density & Molecular structure \\
\hline Tween 80 & Non-ionic & $\mathrm{C}_{64} \mathrm{H}_{124} \mathrm{O}_{26}$ & 1309.63 & 0.016 & 1.064 & \\
\hline Sodium dodecyl sulfate & Anionic & $\mathrm{C}_{12} \mathrm{H}_{25} \mathrm{NaO}_{4} \mathrm{~S}$ & 288.38 & 2.3 & - & \\
\hline Alkylbenzyldimethylammonium chloride & Cationic & $\mathrm{C}_{21} \mathrm{H}_{38} \mathrm{CIN}$ & 339.99 & 2.1 & 0.98 & \\
\hline
\end{tabular}


Table 3

Determination of the experimental error of the analytical technique.

\begin{tabular}{|c|c|c|c|c|c|c|}
\hline & \multirow[b]{2}{*}{ Sample } & \multirow[b]{2}{*}{$\begin{array}{l}\text { PHE initial } \\
\text { in soil / mg }\end{array}$} & \multicolumn{3}{|c|}{ After washing process } & \multirow[b]{2}{*}{ Error $/ \%$} \\
\hline & & & $\begin{array}{l}\text { PHE Liquid } \\
\text { phase /mg }\end{array}$ & $\begin{array}{l}\text { PHE solid } \\
\text { phase/ mg }\end{array}$ & $\begin{array}{c}\text { PHE calculated } \\
\text { by balance }\end{array}$ & \\
\hline \multirow{3}{*}{$\begin{array}{c}\text { Portion } \\
1\end{array}$} & 1 & 3.93 & 3.66 & 0.26 & 3.91 & 0.27 \\
\hline & 2 & 4.17 & 3.97 & 0.45 & 4.42 & 5.86 \\
\hline & 3 & 3.64 & 3.07 & 0.28 & 3.36 & 7.71 \\
\hline \multirow{3}{*}{$\begin{array}{c}\text { Portion } \\
2\end{array}$} & 1 & 4.12 & 3.06 & 0.66 & 3.71 & 9.79 \\
\hline & 2 & 4.08 & 3.17 & 0.15 & 3.33 & 18.37 \\
\hline & 3 & 4.17 & 3.79 & 0.19 & 3.98 & 4.66 \\
\hline \multirow{3}{*}{$\begin{array}{c}\text { Portion } \\
3\end{array}$} & 1 & 3.91 & 3.17 & 0.15 & 3.32 & 15.01 \\
\hline & 2 & 4.16 & 3.47 & 0.22 & 3.69 & 11.29 \\
\hline & 3 & 4.31 & 3.45 & 0.22 & 3.67 & 14.88 \\
\hline \multirow{3}{*}{$\begin{array}{c}\text { Portion } \\
4\end{array}$} & 1 & 4.29 & 3.38 & 0.60 & 3.98 & 7.29 \\
\hline & 2 & 4.16 & 3.55 & 0.43 & 3.98 & 4.38 \\
\hline & 3 & 4.26 & 3.46 & 0.34 & 3.81 & 10.72 \\
\hline & & & & \multicolumn{2}{|c|}{ Average error } & 9.18 \\
\hline
\end{tabular}




\section{Figure captions}

Fig. 1. Time effects in the phenanthrene solubilization with three surfactants. Initial condition was $15 \mathrm{~g}$ spiked kaolinite with $200 \mathrm{ml}$ surfactants (concentration 1\%wt), phenanthrene concentration $500 \mathrm{mg} \mathrm{kg}^{-1}$. Anionic Surfactant, - Cationic Surfactant, $\Delta$ Non-ionic Surfactant.

Fig. 2. Surfactant concentration effects in the phenanthrene solubilization. Initial condition was $15 \mathrm{~g}$ spiked kaolinite with $200 \mathrm{ml}$ surfactants, time $6 \mathrm{~h}$, and phenanthrene concentration $500 \mathrm{mg} \mathrm{kg}^{-1}$. $\square 0.5 \% \mathrm{wt}, \square 1 \% \mathrm{wt}, \square 2 \% \mathrm{wt}$.

Fig. 3. Effects of the initial phenanthrene concentration in the washing process. Initial condition was $15 \mathrm{~g}$ spiked kaolinite with $200 \mathrm{ml}$ surfactants concentration (1\%wt), operation time 6 h. $\square$ Cationic Surfactant, $\square$ Non-ionic, $\square$ Anionic Surfactant, $\square$ Nonsurfactant.

Fig. 4. Aluminium concentration effects in the COD removal in a chemical coagulation test. Initial condition was Surfactant $1 \%$ wt. Phenanthrene $500 \mathrm{mg} \mathrm{kg}^{-1}$. Anionic

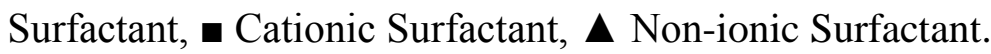

Fig. 5. Aluminium concentration effects in $\mathrm{pH}$ and zeta potential in the treatment of water polluted with anionic surfactant by means of chemical coagulation process. Initial condition was Surfactant $1 \%$ wt. Phenanthrene $500 \mathrm{mg} \mathrm{kg}^{-1}$. pH, — Zeta potential. 
Fig. 6. Aluminium concentration effects in the COD removal in the treatment of water polluted with anionic surfactant and phenanthrene by means of chemical coagulation process. Initial condition was $\bullet$ Surfactant $2 \%$ wt. Phenanthrene $5000 \mathrm{mg} \mathrm{kg}^{-1}$, Surfactant $1 \%$ wt. Phenanthrene $5000 \mathrm{mg} \mathrm{kg}^{-1}$, $\boldsymbol{\Delta}$ Surfactant $1 \%$ wt. Phenanthrene 500 mg kg${ }^{-1}$ * Surfactant $1 \%$ wt. Phenanthrene $0 \mathrm{mg} \mathrm{kg}^{-1}$.

Fig. 7. Aluminium concentration effect in the turbidity removal in the treatment of water polluted with anionic surfactant and phenanthrene by means of chemical coagulation process. Initial condition was $\boldsymbol{\Delta}$ Surfactant $1 \%$ wt. Phenanthrene $500 \mathrm{mg}$ $\mathrm{kg}^{-1}, \mathbf{x}$ Surfactant $1 \%$ wt. Phenanthrene $0 \mathrm{mg} \mathrm{kg}^{-1}$.

Fig. 8. Aluminium concentration effects in the COD removal (a), $\mathrm{pH}(\mathrm{b})$ and Zeta potential (c) in the treatment of water polluted with anionic surfactant and phenanthrene with different initial $\mathrm{pH}$ values by means of chemical coagulation processes. Initial condition was Surfactant $1 \%$ wt. Phenanthrene $500 \mathrm{mg} \mathrm{kg}^{-1}$. Acid initial pH, a Neutral initial $\mathrm{pH}, \boldsymbol{\Delta}$ Alkaline initial $\mathrm{pH}$.

Fig. 9. Metal concentration effects in the COD (a) and turbidity (b) removal in the treatment of water polluted with anionic surfactant and phenanthrene by means of chemical coagulation process using different coagulants. Initial condition was Surfactant $1 \%$ wt. Phenanthrene $500 \mathrm{mg} \mathrm{kg}^{-1}$. $\mathrm{Al}$, - Fe/Al $(80 \% \mathrm{Al}), \boldsymbol{\Delta} \mathrm{Fe} / \mathrm{Al}(50 \%$ $\mathrm{Al}),+\mathrm{Fe} / \mathrm{Al}(20 \% \mathrm{Al}),{ }^{*} \mathrm{Fe}$. 


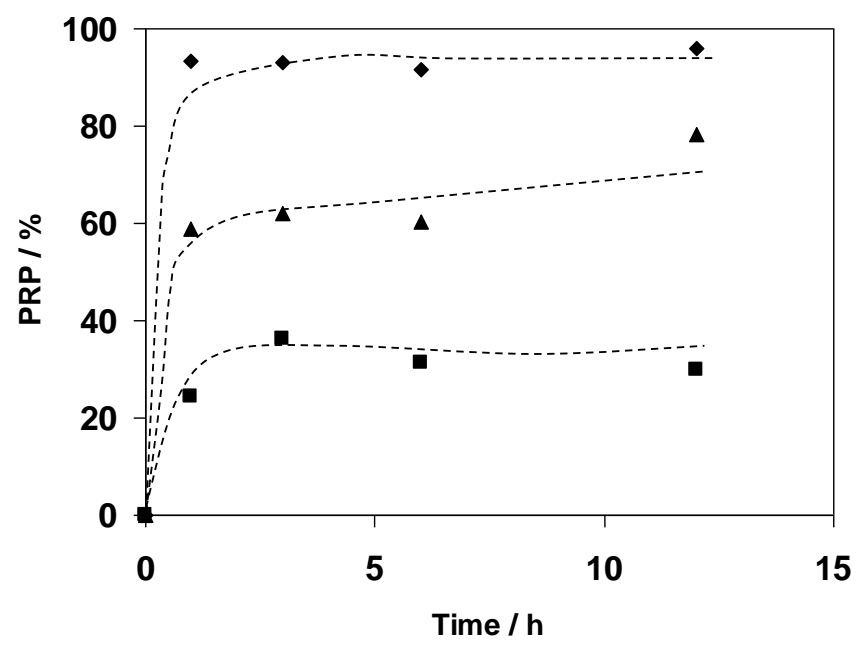

Fig. 1 


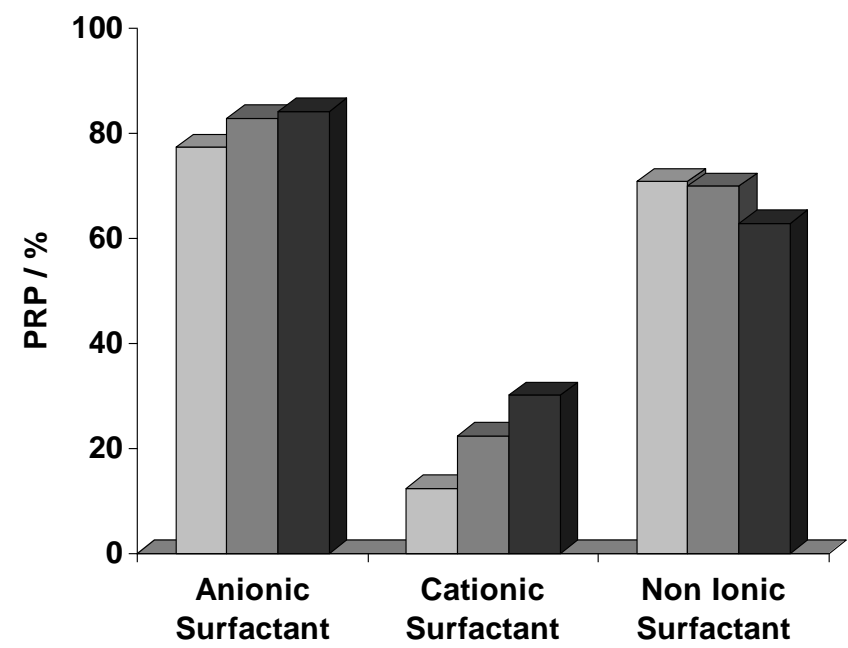

Fig. 2 


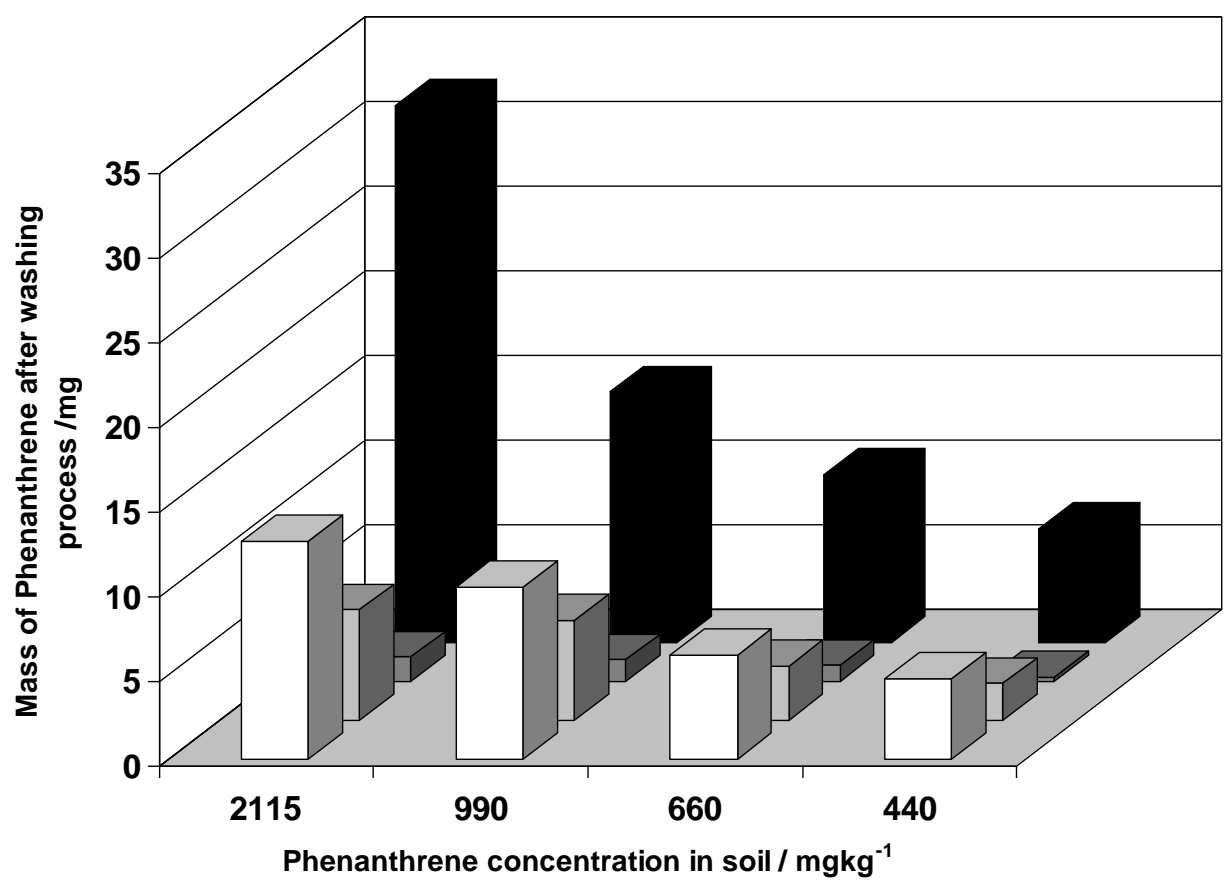

Fig. 3 


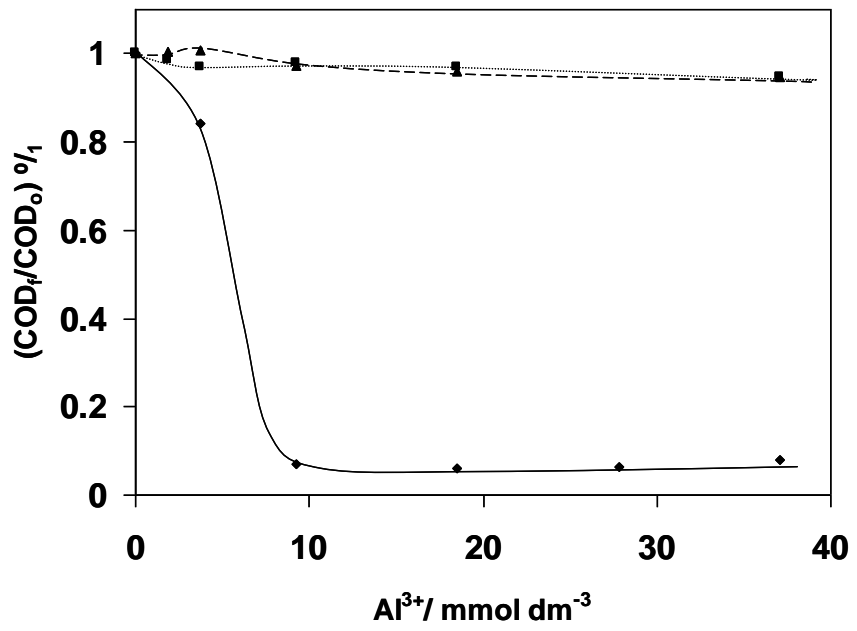

Fig. 4 


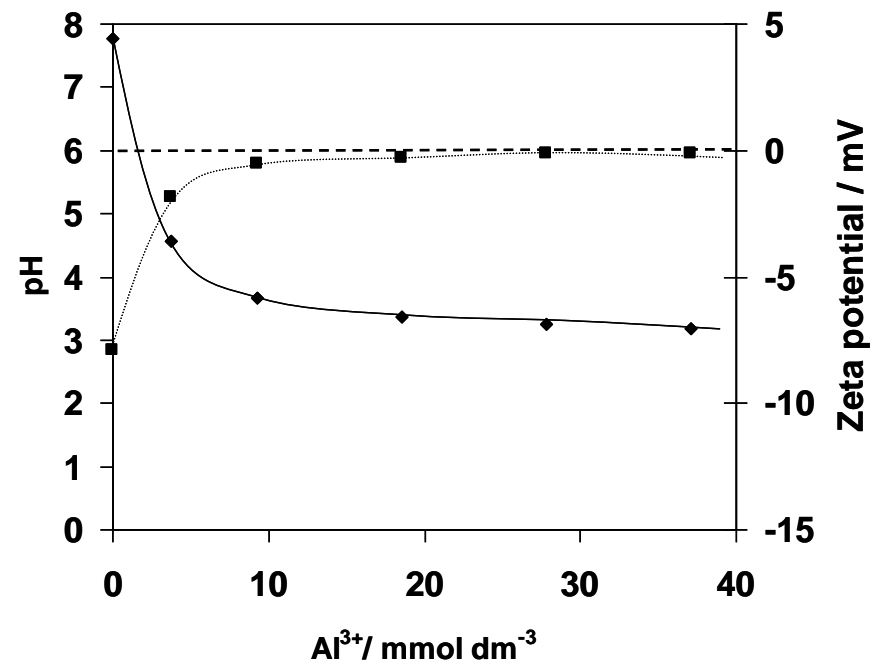

Fig. 5 


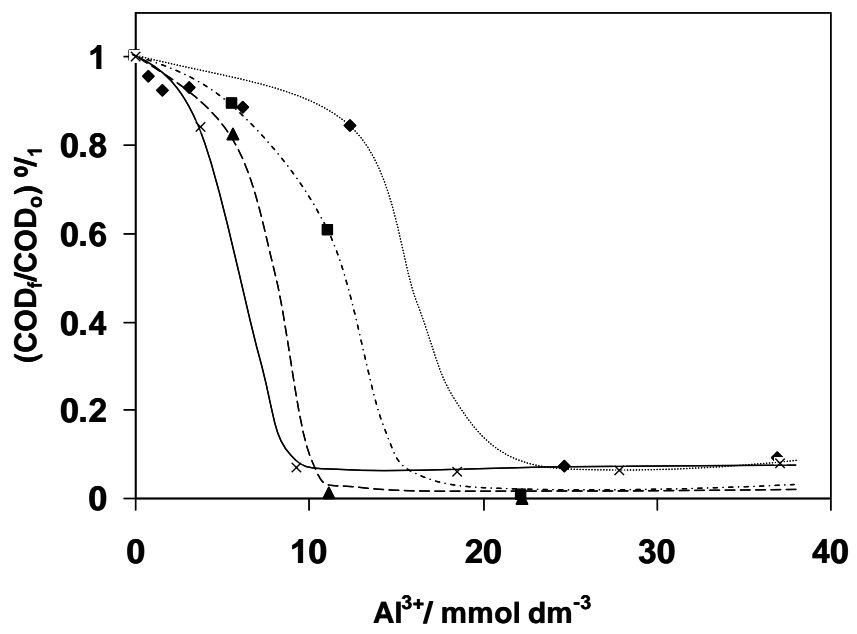

Fig. 6 


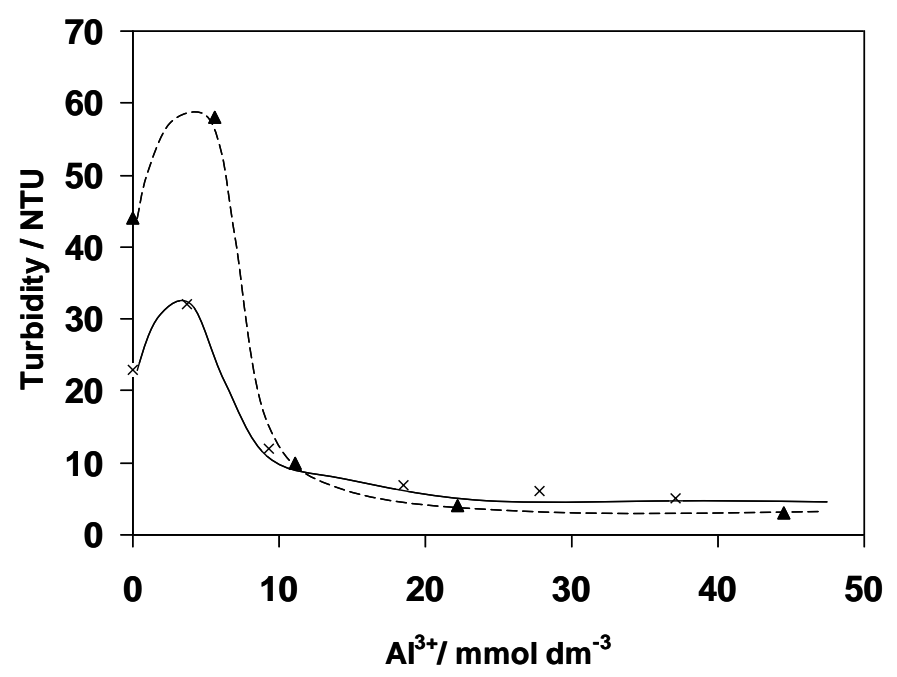

Fig. 7 

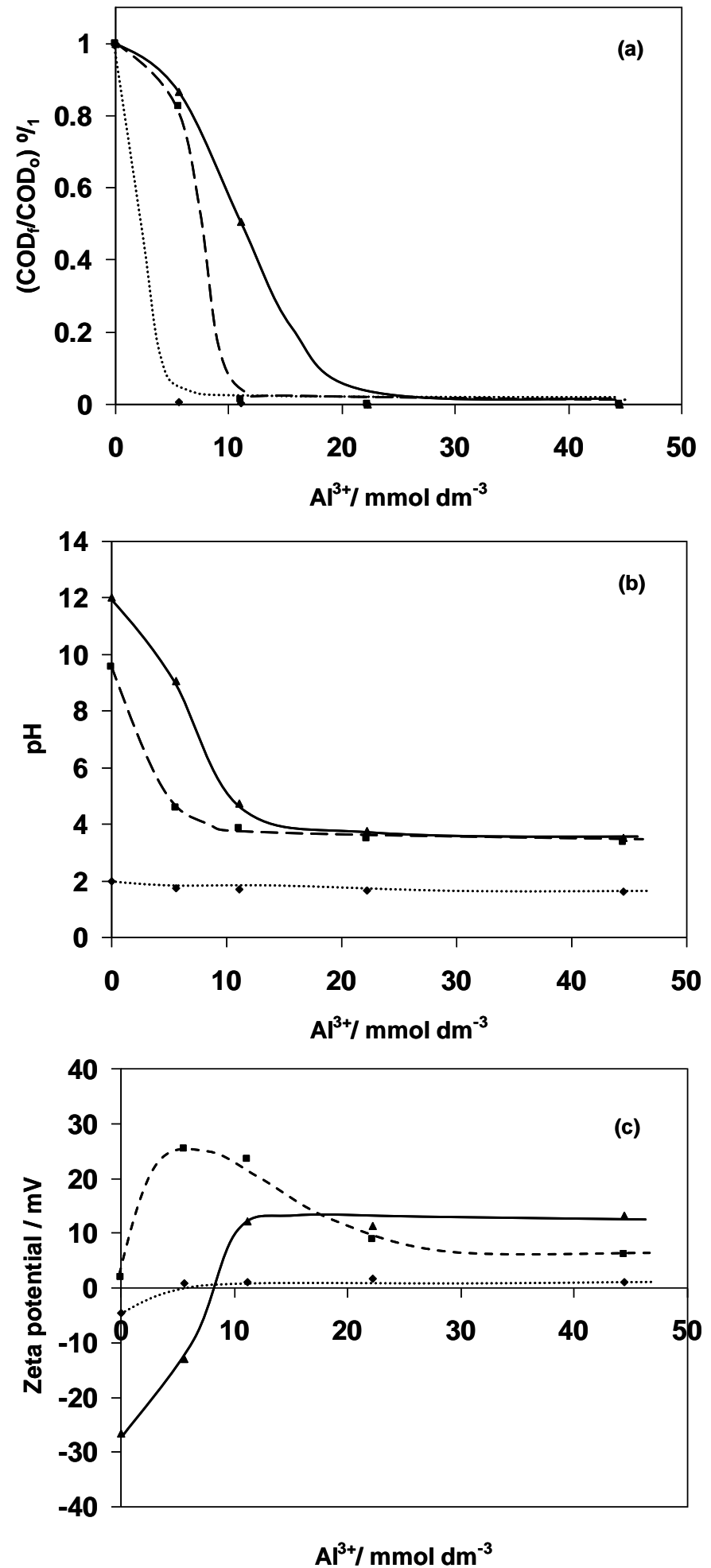

Fig. 8 

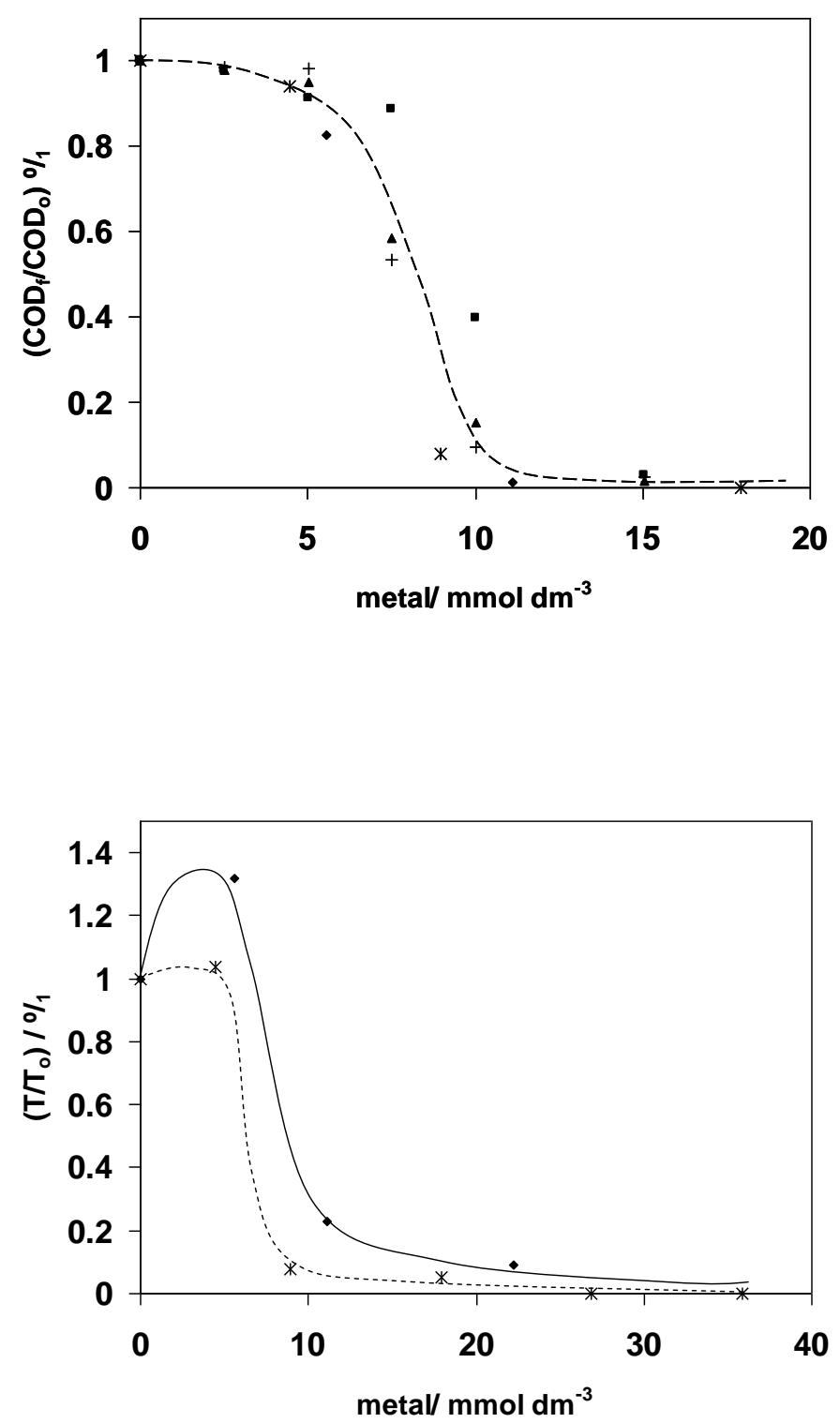

Fig. 9 